\title{
Performance in differential instrumental conditioning as a function of the pattern of partial $S+$ reward*
}

\author{
JAMES H. MCHOSE and DAVID R. BLACKWELL \\ Southern Illinois University at Carbondale, Carbondale, Illinois 62901
}

\begin{abstract}
A total of 46 rats, distributed across two experiments, received differential instrumental conditioning trials in a nonchoice brightness-discrimination apparatus. In each experiment, groups differed with respect to the pattern of rewarded and nonrewarded $S+$ trials. Response in $S-$ was never reinforced. The results of both experiments indicated that the pattern of $S+$ reward events influences $S$ - response levels in a fashion consistent with expectations derived from stimulus-specificity theory.
\end{abstract}

In differential instrumental conditioning, the performance of rats to the stimulus associated with the smaller of two rewards, $\mathrm{S}_{-}$, is depressed relative to that of nondiscrimination $\mathrm{Ss}$ which receive that small reward in both St and S- (cf. Black, 1968; McHose, 1970). This response depression is commonly viewed as indicative of the operation of some inhibitory process much like that involved in the extinction of a simple instrumental response. Perhaps most familiar among such frameworks is the assumption that $\mathrm{S}$ - depression results from the inhibitory effects of frustration as it occurs in $\mathrm{S}-$, frustration engendered by small $\mathrm{S}-$ rewards in the context of larger ( $\mathrm{S}+$ ) rewards (cf. Amsel, 1967). The general viewpoint that $S$ - responding and extinction behavior are functionally interrelated phenomena is supported by the observation that the effects of $S+$ reinforcement parameters on $S$ - behavior are analogous to the effects of acquisition reinforcement parameters on extinction responding. Thus, Sperformance decreases as $\mathrm{S}+$ reward increases (cf. Black, 1968; McHose, 1970) and extinction responding decreases as acquisition reward magnitude increases (e.g., Ison \& Cook, 1964). Similarly, partial reward in acquisition elevates extinction responding and partial $\mathrm{S}+$ reward elevates S- responding (McHose \& Peters, 1973).

In view of the theoretical and empirical precedents for viewing $\mathrm{S}$ - and extinction behavior as interrelated phenomena, and the fact that the specific pattern of partial reward administered in acquisition has profound effects on the extinction of an instrumentally conditioned responses (Capaldi, 1967, 1971, 1972), it would seem reasonable to assume that the pattern of partial $S+$ reward would affect $S-$ performance in differential conditioning. There would appear to be no evidence directly relevant to this assumption, though there are data implicating the sequence in which $\mathrm{S}+$ and $\mathrm{S}$ - trials are administered as an important determinant of S- behavior (Campbell \& Meyer, 1971; Meyer \& Campbell, 1973). The present paper reports two experiments, each of which replicates in substance the

*This research was supported in part by research grant GB30510 from the National Science Foundation. results of two other experiments conducted in our laboratory, which implicate the pattern of partial S+ reward as a determinant of $\mathbf{S}$ - performance.

\section{METHOD}

\section{Subjects}

The Ss in Experiments I and II were 16 and 30 experimentally naive male albino rats, 90 days old, ob tained from the Holtzman Company, Madison, Wisconsin.

\section{Apparatus}

The apparatus consisted of a midgray startbox, $30 \mathrm{~cm}$ long and two parallel $205-\mathrm{cm}$-long alley goal sections, either of which could be aligned with the startbox. One alley goal section was painted flat black and the other was flat white. The interior width and height of each section were $9 \mathrm{~cm}$ and $10 \mathrm{~cm}$, respectively. A solenoid-operated opaque door separated the start and alley sections of the apparatus, and translucent retrace doors, normally open, separated the alley from the goal sections. Photcell-clock circuitry provided traversal times over the first two $60-\mathrm{cm}$ segments of the alley, yielding start and running times. A Model 100A Pamotor fan, mounted on the exterior wall of the goal segment of the apparatus exhausted air from the apparatus via holes in the goalbox wall.

\section{Procedure}

In both Experiments, Ss were placed on 23-h food deprivation regimen 10 days prior to the first experimental day (Day 11). On Days 9 and $10,1 \mathrm{~g}$ of $45-\mathrm{mg}$ Noyes pellets, identical to the subsequent reinforcement pellet, was incorporated in Ss' daily ration, Ss were handled for approximately $5 \mathrm{~min}$ each, and allowed to explore the start and alley sections of the apparatus for approximately $2 \mathrm{~min}$.

In Experiment I, all Ss received two S+ (e.g., black alley) and two $S-$ trials per day for 28 days. One $S+$ trial per day was rewarded ( 20 pellets), while $S$ - trials were always nonreinforced. Two randomly constituted groups of eight $S$ s each differed with respect to the order of reward $(R)$ and nonreward $(N)$ events on $\mathrm{S}+$ trials each day. Group $\mathrm{R}-\mathrm{N}$ always received an $\mathrm{R}$-event on the first $S+$ trial of the day and an $N$ event on the second $S+$ trial, while for Group N-R this order was reversed. The brightness of $\mathrm{S}+$ was counterbalanced between Ss within each group. Black (B) and white (W) trials were administered according to the following repeating cycle: BWBW, WBWB, BBWW, WWBB, WBBW, and BWWB.

In Experiment II, all $S$ s received three $S+$ and one $S-$ trial each day for 18 days. Two $S+$ trials were rewarded (10 pellets) while $\mathrm{S}$ - trials were always nonrewarded. Three randomly constituted groups of $10 \mathrm{Ss}$ each differed with respect to the 
pattern of $\mathrm{R}$ and $\mathrm{N}$ events obtained on $\mathrm{S}+$ trials each day; Groups RNR, RRN, and NRR. Trials were administered according to the following recursive cycle of $S+(P)$ and $S-(M)$ : PPMP, PMPP, MPPP, PPPM. The brightness of $\mathrm{S}+$ was counterbalanced within each group.

Trials were administered to squads of eight $\mathrm{Ss}$ in Experiment I, two from each of the four conditions (including the brightness counterbalance factor) and to squads of six Ss in Experiment II, one from each of the six conditions. Each $\mathrm{S}$ within a squad received its first daily trial before any $S$ received its second daily trial, etc. This procedure resulted in an intertrial interval of approximately $6 \mathrm{~min}$ in each experiment. On each trial, the start door was opened after $S$ had oriented toward the door for $3 \mathrm{sec}$, and $\mathrm{S}$ was removed from the goalbox immediately after eating, or on $\mathrm{N}$ trials, after $6 \mathrm{sec}$.

\section{RESULTS}

Since the two response measures recorded yielded essentially redundant information, only the run measure will be presented. Figure 1 presents group mean run speeds as a function of blocks of 8 total trials for the groups of Experiment I. For both groups, speeds on $\mathbf{S}+$ trials are plotted separately from those on $S-$ trials. For Group R-N only, speeds on S- trials are further separated according to whether that trial was the first or second S- trial of the day. As may be seen, both groups eventually developed a discrimination, running faster on $\mathrm{S}+$ than on $\mathrm{S}-$ trials. Moreover, the pattern of $\mathrm{S}+$ reinforcement influenced $S-$ behavior, Group R-N running slower to $S-$ than did Group N-R. Finally, Group R-N ran more slowly on the second $S$ - trial of the day than on the first daily $\mathrm{S}$ - trial. Analysis of

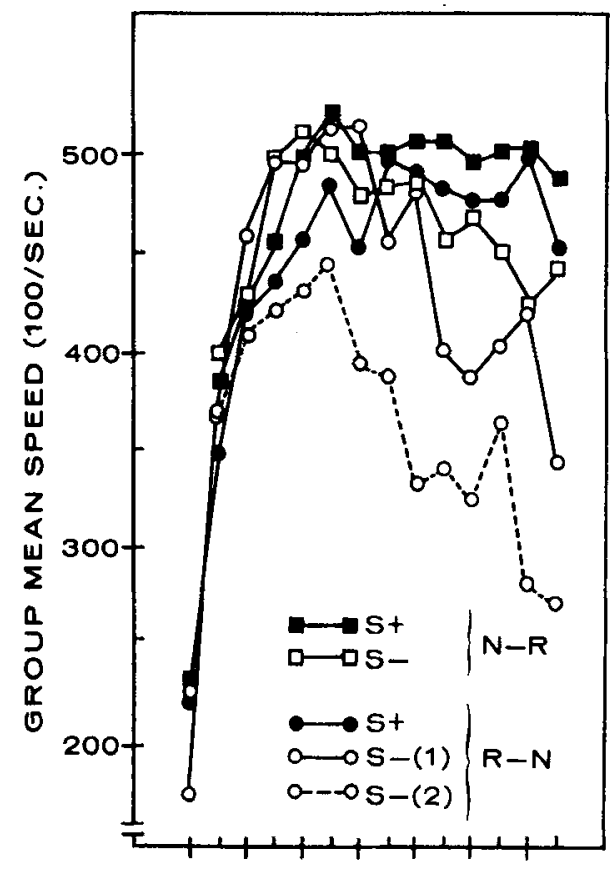

TRIAL BLOCKS

Fig. 1. Group mean running speeds as a function of blocks of eight total trials in Experiment $I$. variance of the data over Blocks 11-14 including groups, S+ brightness, discriminanda, and trial position (first or second trial of the day) as factors, indicated that each of the aforementioned effects were statistically reliable. This analysis yielded a significant Groups by Discriminanda by Trial Position interaction $(F=10.82$, $\mathrm{df}=1 / 12, \quad \mathrm{p}<.01) . \quad$ Subsequent within-S pair comparisons (Tukey HSD) indicated that, for Group N-R, S+ $(1$ and 2$)$ speeds were reliably $(\alpha<.05)$ faster than $S-(1$ and 2 ) speeds while, for Group R-N, $S-(1)$ and S- (2) speeds differed from each other and from $S+(1)$ and $S+(2)$ speeds. No other within-Sdifferences approached statistical significance. Between-S comparisons (Tukey a) indicated that the S(1) and S- (2) speeds of Group R-N were reliably $(\alpha=.05)$ depressed relative to those of Group N-R.

Turning to the data of Experiment II, group mean run speeds for each group are plotted as a function of blocks of eight total trials in Fig. 2. For each group, speeds on the six S+ trials within a block are plotted separately from those on the two $\mathrm{S}$ - trials within a block. As may be seen in Fig. 2, each group eventually developed a discrimination, running faster to $S+$ than to $S-$. Variance analysis of the data for Blocks 7-9, including groups, S+ brightness, and discriminanda as factors, yielded a significant discriminandum effect $(F=112$, $\mathrm{df}=1 / 24, \quad \mathrm{p}<.001)$ and a significant Groups by Discriminanda interaction $(F=5.29, \quad d f=1 / 24$, $\mathrm{p}<.025)$. Subsequent paired comparisons ( $t$ tests) indicated that each group ran reliably $(p<.01)$ faster on $\mathrm{S}+$ than on $\mathrm{S}-$ trials.

Of primary interest in Fig. 2 is the indication that Groups RNR and NRR attained a comparable Sperformance level which was considerably slower than that of Group RNN. Paired comparisons (Tukey a) subsequent to the analysis of variance reported above indicated that the $\mathrm{S}-$ performance differences between Groups RNR and RRN, and between Groups NRR and RRN, were statistically reliable $(p<.05)$. No other between-group comparisons of either $\mathrm{S}-$ or $\mathrm{S}+$ performance levels were statistically significant.

\section{DISCUSSION}

The results of ExperimentsI and II clearly indicate that the pattern of partial reward received within S+ influences performance to a nonrewarded $\mathrm{S}-$. Thus, in Experiment I, the Ss which received only R-N trial-to-trial transitions within S+, Group R-N, ran slower to $\mathrm{S}$ - than did Ss which received only N-R transitions. In Experiment II, Groups RNR and NRR ran more slowly to $\mathrm{S}$ - than did Group RRN. In both experiments, several other sequential variables such as the number of within-days transitions from $S+(R)$ to $S-, S+(N)$ to $S-$, $\mathrm{S}-$ to $\mathrm{S}+(\mathrm{R})$, and $\mathrm{S}-$ to $\mathrm{S}+(\mathrm{N})$ were controlled between or among groups and thus the group differences 


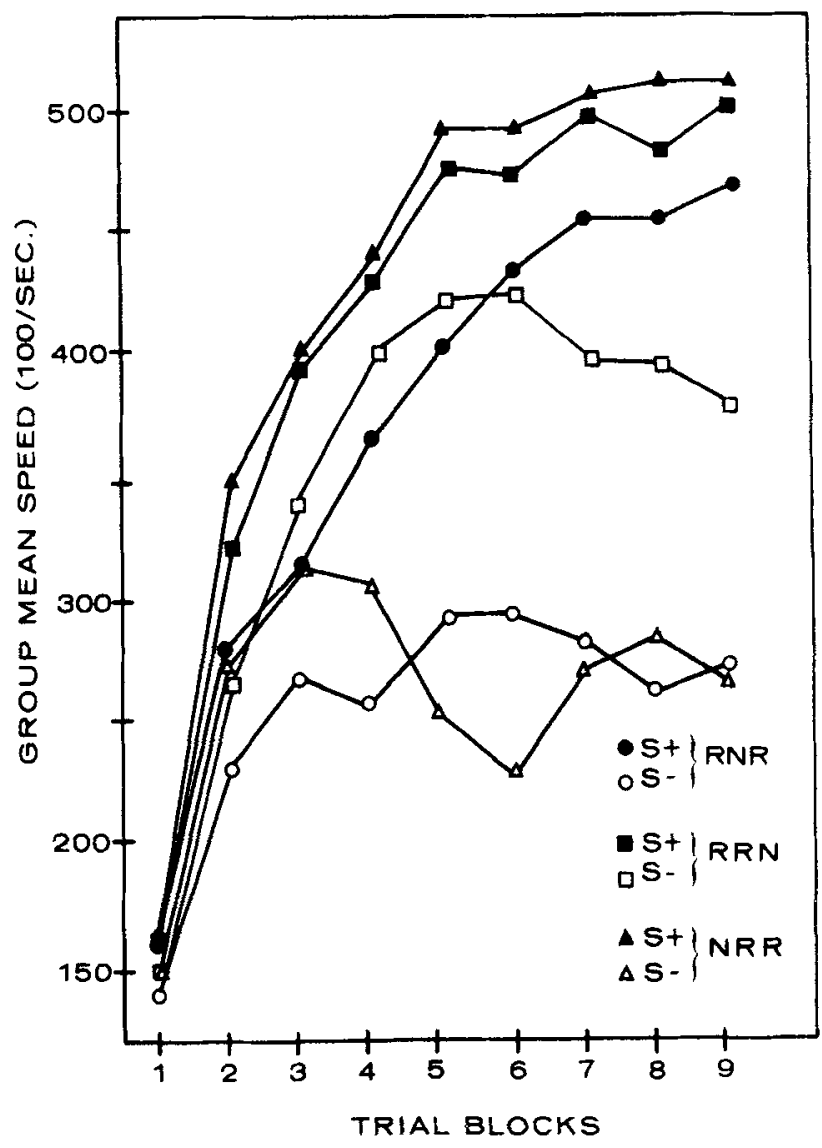

Fig. 2. Group mean running speeds as a function of blocks of eight total trials in Experiment II.

obtained are not attributable to sequential variables elsewhere shown to influence $S$ - responding (Campbell \& Meyer, 1971; Meyer \& Campbell, 1973).

While the results of Experiments I and II are consistent in implicating the pattern of partial S+ reward determinant of $\mathrm{S}-$ behavior, they are seemingly in contradiction with respect to the precise role of N-R or R-N transitions. In Experiment I, the Ss which received only R-N transitions within a day showed a marked depression of $\mathrm{S}$ - performance relative to Ss which received only N-R transitions. In Experiment II, however, the group which received only R-N transitions within a day, Group RRN, displayed faster S- speeds than did groups with only N-R transitions, Group NRR, or with both N-R and R-N transitions, Group RNR. Despite the apparently contradictory findings, the results of the experiment are, at least in part, readily interpretable within the familiar theorectical framework developed to account for the effects of sequential reinforcement variables on resistance to experimental extinction (Capaldi, 1967, 1971, 1972).

Assume with Capaldi that $\mathrm{R}$ and $\mathrm{N}$ events on any one trial occasion different stimuli, $S_{R}$ and $S_{N}$, which are in some fashion reinstated on the next trial, and therefore available for conditioning to the instrumental response on that trial. Assume further that these stimulus traces or memories are stimulus specific (cf. Capaldi, 1972), i.e., in the present context, the reward-related stimulus reinstated on any $S+$ trial is appropriate to the preceding $S+$ reward event, not simply to the preceding trial. Finally, assume that the reward-related stimuli reinstated on any trial differ according to the age of the memory or stimulus trace, i.e., that the stimulus produced by an $\mathrm{N}$ event, for example, varies according to the (intertrial) interval (ITI) between the $\mathrm{N}$ event and its reinstatement. It should be recognized that, in the present context, ITI refers to the interval between like discriminandum presentations.

Applying the assumptions of specificity theory to Experiment I, it should be clear that different stimuli occur on the first and second S-trials of each day. The reward-related stimulus present on the first such trial is an $S_{N}$ associated with a long (24-h) ITI, $S_{N L}$ while on the second $S$ - trial of each day, the relevant stimulus is an $S_{N}$ associated with a shorter (within-day) ITI, $S_{N S}$. Examinations of the reinforcement and discrimindandum schedules administered to Group R-N reveals that while this group acquired no habit strength to $S_{N S}$, it did acquire habit strength to $S_{N L}$ since the first $S+$ trial of each day reinstated $S_{N L}$ and response was reinforced in the presence of this stimulus. The observation that Group R-N ran faster on the first S-trial of each day (to $S_{N L}$ ) than on the second daily $S$ trial (To $S_{N S}$ ) is thus entirely consistent with a stimulus-specificity analysis. Since Group N-R accrued habit strength to $\mathrm{S}_{\mathrm{N}}$ on the second $\mathrm{S}+$ trial of each day, its performance to this stimulus when reinstated on the second $S$ - trial of each day should be above that of Group R-N. Again, this expectation is confirmed by the data.

While certain portions of the data of Experiment $I$ are consistent with a stimulus specificity analysis, such an analysis is not without some apparent deficiencies. Thus, it would seem that since Group N-R acquired no habit to $S_{N L}$, it should have displayed slower speeds on the first as compared with the second $\mathrm{S}-$ trial of each day, speeds as slow, in fact, as those evidenced by Group R-N on the second $\mathrm{S}$ - trial. Note, however, that the assumptions of specificity theory which have been applied to the present data do not exhaust the assumptions contained in this theory as outlined elsewhere (Capaldi, 1967). Such variables as N length, for example, could well influence the behavior of Groups N-R and R-N, although the application of an $\mathrm{N}$-length principle in the present context would required a more detailed account of how the N-length stimuli are modified when successive $\mathrm{N}$ events occur at different ITIs than is presently available. At this juncture, the data of Experiment I are merely promising with respect to an eventual stimulus-specificity theory analysis of $\mathrm{S}$ performance. The data of Experiment II are even more promising in this regard. 
In Experiment II, all groups received only one S- trial per day. On $S-$ trials, then, Ss were required to respond in the presence of $S_{N L}$. While Groups RNR and NRR were rewarded each day for responding to $S_{N S}$, they acquired no habit strength to $S_{N L}$. Group RRN, however, acquired habit strength to $S_{N L}$, on the first daily $\mathrm{S}+$ trial, but not to $\mathrm{S}_{\mathrm{NS}}$. The observation of comparable S- performance levels for Groups RNR and NRR, levels below that of Group RRN, is thus consistent with stimulus-specificity theory. And the apparent contradiction between the results of the two experiments with respect to the role of R-N and N-R transitions is thus, in large part, resolved by a stimulus-specificity analysis.

Note that the preceding analyses assume that $S_{N}$ is reinstated on any trial if the last response in the same discriminandum was nonrewarded. Apparently this assumption should be limited to experiments in which the reinforcement contingencies differentially associated with $\mathrm{S}+$ and $\mathrm{S}-$ generate incentive values sufficiently different to provide for differential responding to $S+$ and $\mathrm{S}-$. Thus, the assumption is useful for a sequential analysis both of the present data and of those reported by Capaldi (1972), data from experiments in which S+ and $\mathrm{S}$ - incentive values were substantially different (cf. McHose, Maxwell, \& McHewitt, 1971; McHose \& Peters, 1973). However, when $S+$ and $S-$ incentive values are minimally different, the alternative assumption that $S_{N}$ is reinstated if the preceding trial was nonrewarded seems most useful in a sequential analysis of $\mathrm{S}$ (extinction) responding (cf. Mellgren $\&$ Dyck, 1972).

Collectively, the results of Experiments I and II are consistent with the general viewpoint that at an ITI of approximately $6 \mathrm{~min}$ performance to a nonrewarded stimulus in differential instrumental conditioning is influenced by the same behavioral processes thought to affect extinction responding. More specifically in this context, the present results are for the most part supportive of sequential or stimulus-specificity analyses (Capaldi, 1967, 1971, 1972) as developed to interpret extinction phenomena. Finally, while the present data do indeed suggest that $\mathrm{S}-$ performance depends in part on the processes stipulated within sequential theory, such an observation should not be made without some constraints. For example, the reliable observation that the S- performance of discrimination Ss is depressed relative to that of a condition which always receives the S- reward (cf. Black, 1968; McHose, 1970) does not appear to follow from any combination of stimulus-specificity assumptions as applied to the present data.

\section{REFERENCES}

Amsel, A. Partial reinforcement effects on vigor and persistence. In K. W. Spence and J. T. Spence (Eds.), The psychology of learning and motivation. New $w^{2}$ York: Academic Press, 1967.

Black, R. W. Shifts in magnitude of reward and contrast effects in instrumental and selective learning: $A$ reinterpretation. Psychological R eview, 1968, 75, 114-126.

Campbell, E. M., \& Meyer, P. A. Effects of daily reward sequence on simultaneous and successive negative contrast in rats. J ournal of Comparative \& Physiological Psychology, $1971,74,434-440$.

Capaldi, E, J. A sequential hypothesis of instrumental learning. In K. W. Spence and J. T. Spence (Eds.), The psychology of learning and motivation. Vol. 1. New York: Academic Press, 1967.

Capaldi, E. J. Memory and learning: A sequential view point. In W. K. Ho nig and P. H. R. James (Eds.), A nimal memory. Academic Press, 1972.

Capaldi, E, J., \& Morris, M. D. Reward schedule effects in extinction: Intertrial interval, memory, and memory retrieval. Learning \& Motivation, in press.

Ison, J. R., \& Cook, P. E. Extinction performance as a function of incentive masnitude and number of acquisition trials. Psychonomic Science, 1964, 1, 245-246.

McHose, J. H. Relative reinfor cement effects: $S_{1} / S_{2}$ and $S_{1} / S_{1}$ Paradigms in instrumental conditioning. Psychological R eview, $1970,77,135-146$.

McHose, J. H., Maxwell, F. R., \& McHewitt, E. R. The role of nonreward in $S+$ and in $S-$ in differential conditioning. Journal of Experimental Psy chology, 1971, 88, 282-284.

McHose, J. H., \& Peters, D. P. Differential instrumental conditioning as a function of the percentage and amount of $S+$ reward. Journal of Experimental Psychology, 1973, 100, 413-415.

Meligren, R. L., \& Dyck, D. G. Partial reinforcement effect, reverse partial reinforcement effect, and generalized partial reinforcement effect within subjects. Journal of Experimental reinforcement effect within sub
Psychology, 1972, 92, 339-346.

Meyer, P. A., \& Campbell, E. M. Role of daily reward sequences on $\mathbf{S}$ - discrimination contrast in rats. Journal of Comparative \& Physiological Psychology, 1973, 82, 426-433.

(Received for publication A pril 4, 1974; revision accepted July $17,1974$. ) 